\title{
Max-Min Fairness in WMNs with Interference Cancelation Using Overheard Transmissions
}

\author{
Mateusz Żotkiewicz \\ Institute of Telecommunications, Warsaw University of Technology, Nowowiejska 15/19, 00-665 Warszawa, Poland \\ Correspondence should be addressed to Mateusz Żotkiewicz; mzotkiew@tele.pw.edu.pl
}

Received 28 February 2014; Accepted 24 April 2014; Published 13 May 2014

Academic Editor: Dritan Nace

Copyright (C) 2014 Mateusz Żotkiewicz. This is an open access article distributed under the Creative Commons Attribution License, which permits unrestricted use, distribution, and reproduction in any medium, provided the original work is properly cited.

We show an impact of using interference cancelation mechanisms for signals that have been overheard in the past on performance of fair wireless mesh networks. In our research we show that even in those very restricted conditions and max-min cost function, the idea of interference cancelation can significantly increase the capacity of such networks. In order to approximate possible advantages of using interference cancelation in the considered conditions, we propose a novel MIP model that allows for calculating perfect scheduling and maximal throughput in a network. We compare the results with cases when the interference cancelation mechanisms are disabled. Our results show that using interference cancelation mechanisms for signals that have been overheard in the past increases a network throughput by $40 \%$ on average in approximately $20 \%$ of test cases.

\section{Introduction}

Mesh networking in wireless communications is a way to increase network capacity, for example, with respect to singlehop cellular networks, through using short-distance radio links of relatively high capacity and establishing point-topoint connections on multi-hop routes. In this way packets from an origin are relayed by intermediate nodes on their way to a destination. Today, wireless mesh networks (WMN) are mainly used for providing affordable Internet access to communities of users in metropolitan areas (see $[1,2])$. A typical wireless access network (WAN) is based on the IEEE 802.11-family Wi-Fi standards and consists of a set of fixed mesh nodes - routers and Internet gateways-interconnected by radio links. The mesh routers serve mesh clients (which are either fixed or mobile) and at the same time perform the packet relay function. WMN is a cost-efficient off-the-shelf networking that provides bandwidth in the range of up to hundreds Mbps. For popular surveys on WMN, see $[3,4]$.

Wireless PHY layer techniques are very mature nowadays and can achieve close to Shannon limit transmission capacities in point-to-point scenarios. Therefore, novel research directions that can result in the capacity increase of wireless networks are of interest now. One of such directions is to increase the spatial reuse by increasing a number of simultaneous transmissions that use the same medium. The direction proved to be very promising when IEEE 802.11n enhancements for higher throughput amendment [5] started to be widely used and a throughput of a single Wi-Fi transmission increased from $54 \mathrm{Mbps}$ to $600 \mathrm{Mbps}$, mostly due to introduction of multiple-input multiple-output (MIMO) techniques.

In MIMO a source node transmits a number of independent data streams using a number of different antennas. In a sink node the streams are received also using a number of antennas. Although the streams are transmitted simultaneously and they strongly interfere with each other, they still can be successfully decoded due to phase shifts between them while being received by different antennas of the sink node.

The idea of using receiving techniques that involve recognizing and removing interferences from a signal is now shifting from a point-to-point scenario (like in MIMO) to more general scenarios like concurrent transmissions of many independent nodes. The technique is called interference cancelation and has been successfully implemented, for instance, in cellular networks [6]. A recent survey on successful interference cancelation implementations can be found in [7]. Currently, possibilities of employing the techniques 
in wireless mesh networks along with other ideas like zerocanceling [8] or interference alignment $[9,10]$ are being investigated.

One of assumptions of majority of work on interference canceling in wireless mesh networks is that canceling mechanisms are perfect. Therefore, if an interfering signal can be decoded, then it can be completely removed. The assumption works well when acceptable signal-to-interference-plus-noise (SINR) ratios of used modulations and codings schemes (MCSs) are rather low. When we assume that utilized MCSs require much higher SINR thresholds, then the quality of the interference canceling mechanisms becomes a factor and the previously justified assumption of perfect cancelation should be reconsidered.

In this paper we consider a case when SINR thresholds are relatively high and the usage of interference cancelation mechanisms is restricted only to cases when an interfering signal is canceled not because it can be decoded, but because it had been overheard in the past. In our research we show that even in this very restricted conditions the idea of interference cancelation can significantly increase the capacity of a wireless mesh network. In order to approximate possible advantages of using interference cancelation in the restricted conditions, we propose a novel mixed integer programming (MIP) model that describes the problem and allows for finding a perfect scheduling, thus calculating maximal throughput in a network. We compare the results with results obtained using an MIP model of [11] that describes a case when the interference cancelation mechanisms are not used. Our results show that using interference cancelation mechanisms in the restricted conditions increases a network throughput by $40 \%$ on average in approximately $20 \%$ of test cases.

The paper in organized as follows. In Section 2 we briefly explain technical issues of interference cancelation in overall and special features of the approach used in this research in detail. Next, in Section 3 we present a novel MIP model that allows for optimization of scheduling in wireless mesh networks that use the interference cancelation mechanisms for overheard transmissions. In Section 4, numerical results are presented. They are followed by conclusions given in Section 5.

\section{Interference Canceling}

The core components of a WMN that form its infrastructure/backbone are mesh points (MPs). They are responsible for relaying traffic. They can relay traffic of either other MPs or legacy nodes (STAs). Obviously they can also send their own traffic. Legacy STAs are connected to MAPs (specialized MPs that also act as access points for STAs) and follow the principle of a generic wireless local area network node. Other specialized MPs are responsible for connecting a WMN to an external network (usually the Internet). They are called mesh point portals or gateways and are abbreviated by MPP.

In radio networks two stations $A$ and $B$ can communicate if the power of the signal received from station $A$ at station $B$ in comparison to the noise at station $B$ is greater than an acceptable signal-to-noise (SNR) threshold for the applied modulation and coding scheme (MCS). In WMN networks the radio resources are shared between all MPs. Thus, typically all MPs cannot transmit simultaneously but, of course, simultaneous transmissions by subsets of stations are still possible. In such a case, however, not only the noise can disrupt transmission but also the interferences induced by other MPs. Therefore, in the context of WMN we use the notion of signal-to-interference-plus-noise ratio (SINR) instead of SNR. SINR is understood as a proportion of a received power to a sum of the noise and the received interferences.

As there is little one can do with the noise, novel ways interferences that can be combated are of great interest. Two of such techniques, that is, zero-forcing [8] and interference alignment [9], are successfully used in pointto-point transmissions in MIMO technology. In WMN a promising interference combating strategy consists of various interference cancelation (IC) techniques. The notion behind IC is that if a node receives a signal consisting of an interfering signal $A$ and a signal of interest $B$ (assume that $B$ cannot be decoded due to the interferences) and data encoded in $A$ are known, then the interfering signal $A$ can be reconstructed in the considered node using the known data, subtracted from the received signal, and finally $B$ can be decoded.

The strategy can be used twofold. First, the signal $A$ can be itself strong enough that it can be decoded in the receiving node regardless of interferences created by the signal $B$. Then, having data encoded in $A$, it is possible to reconstruct $A$ and subtract it from the received signal. This approach was used in practice, for instance, in [12] where authors successfully implemented IC in a ZigBee network. However, the approach has one major disadvantage: it proved to work efficiently only with relatively low SINR thresholds. The reason is limited efficiency of IC detectors. In ideal conditions it is possible to reduce an interfering signal by up to $30 \mathrm{~dB}$ [12]. In practice, due to propagation and multipath issues, the efficiency rate is much smaller. Therefore, assuming that an SINR threshold is relatively high, if it is possible to decode an interfering signal $A$, then the signal is too strong to be canceled to such an extent that a signal of interest $B$ can also be decoded. The way such a version of the problem can be approached in terms of optimization can be found in [13].

In our research we concentrate on the second way of using IC (see, for instance, $[14,15])$. In this approach a main assumption is that data carried by an interfering signal $A$ is known from previous transmissions. Consider an example presented in Figure 1, where $a$ wants to send a packet to $c$ and $d$ wants to send a packet to $f$. Both packets are relied, the former by $b$, the latter by $e$. The arrows represent that possible links packets can be sent on; that is, if there is an arrow between a pair of MPs, then packets can be successfully sent between them. What is more, the arrows also represent possible interferences; for example, if $a$ is transmitting to $b$, then $d$ cannot transmit to $e$ as the first transmission will jam the second. Therefore, in order to successfully meet both traffic demands, four time slots have to be used, because, due to possible interferences, there are no pairs of needed transmissions that can be conducted simultaneously. 


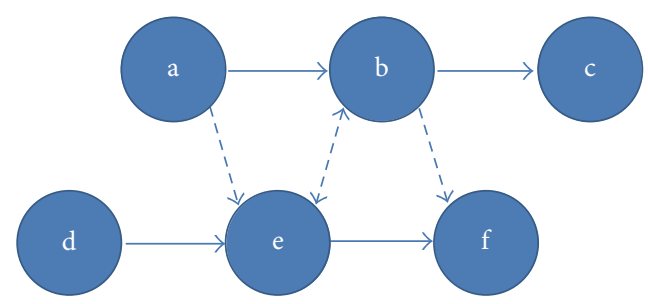

Figure 1: Example of using interference cancelation.

However, when transmissions are properly ordered and IC mechanisms are utilized, the traffic demands can be met in just three time slots. First, $a$ transmits to $b$, but the packet is overheard, that is, successfully received and decoded, by $e$. Then, $b$ transmits to $c$ and simultaneously $d$ transmits to $e$. During this step $e$ receives two signals (from $b$ and from $d$ ) that interfere with each other. However, $e$ knows data encoded in the signal from $b$; thus it can reconstruct it and subtract it from the received signal (perform interference cancelation). Finally, it can decode the packet sent from $d$. In the last step, $e$ sends the decoded packet to $f$, thus satisfying the traffic demands. This simple example (other more sophisticated examples can be found in [16]) illustrates IC mechanisms that are considered in our research.

A vigilant eye would notice that this IC mechanism can be only used when a packet relayed by a node is not subject to any modifications in this node. Obviously that is usually not the case, as the relay node changes both a header and a CRC code that ends the packet. However, when appropriately approached, even this obstacle can be combated. The way of doing this is to let the to be canceled packet by transmitted slightly earlier. If the header of the packet is received by a node that is going to cancel it without any interferences, then the rest of this packet including CRC can be treated with IC mechanisms without any further problems and will not affect other transmissions that are supposed to happen right after the header of the canceled packet is received.

A major novelty of this paper is a tractable optimization model that encompasses the described IC mechanisms. In our research we assume a common interference model where two transmissions interfere only if a sender in the first transmission can successfully transmit to a receiver in the second transmission, or a sender in the second transmission can successfully transmit to a receiver in the first transmission. More sophisticated interference model, where a signal power is taken into account and all concurrent transmission interacts with each other, can be found, for instance, in [17].

We consider an architecture presented in Figure 2, where a set of MPs, a set of MPPs, and a set of links, routing, and possible interferences are given, and our task is to schedule transmissions in an optimal way. As volumes of demands in WMN are hard to predict, we decided to use the maxmin fairness (MMF) paradigm (see, for instance, [18]) and maximize the throughput of the worst served MP.

We assume that the considered WMN is synchronized; a number of time slots are infinitely repeated. Our task is to assign point-to-point transmissions to those time slots in

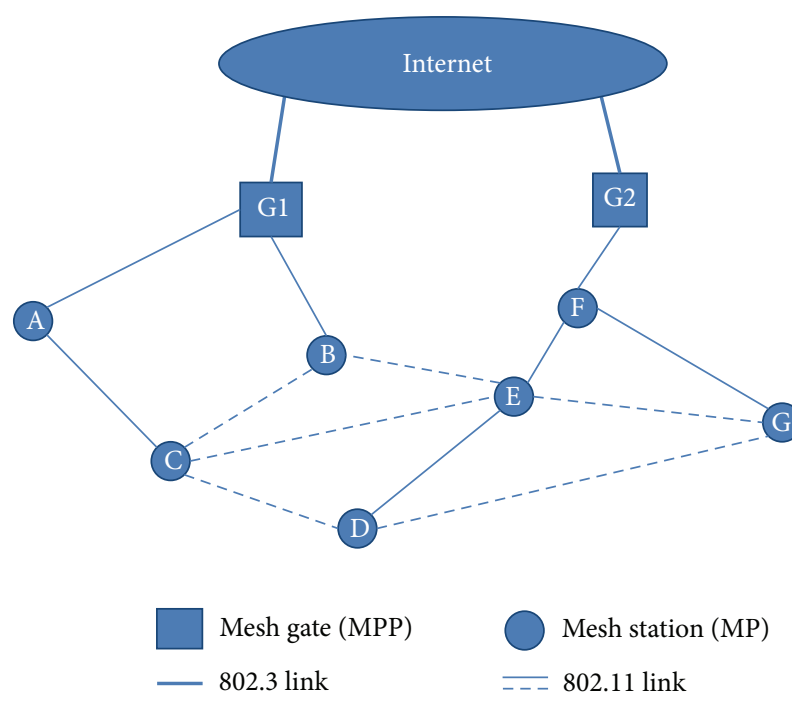

FIGURE 2: Wireless mesh network architecture.

such a way that the objective is maximized. In order to better understand the notion of time slots, consider the example of Figure 1 with demands from $a$ to $c$ and from $d$ to $f$ and a bit rate of each link equal to $1 \mathrm{Mbps}$. If there are four available time slots and the IC mechanisms are disallowed, then both $a$ and $d$ will be the worst served MPs with $0.25 \mathrm{Mbps}$ for each of them. What is more, when the IC mechanisms are used, the situation will not change. Although only three time slots are needed to serve each relevant MP with $0.25 \mathrm{Mbps}$, the fourth time slot cannot be effectively used; thus it is wasted in every cycle. On the other hand, when there are six available time slots and the IC mechanisms are disallowed, then only $0.17 \mathrm{Mbps}$ can be provided to the worst served MP, while the other MP can be provided with $0.33 \mathrm{Mbps}$. However, when the IC mechanisms are used, both MPs can be served with $0.33 \mathrm{Mbps}$.

There is substantial literature on the optimization of scheduling and MCS selection in wireless mesh networks (see, for instance, [11, 17] and references therein). However, the topic of the scheduling and MCS selection optimization under IC constraints has not been yet exhaustively researched. In [19] the problem is raised, but the authors do not take routing into account. On the other hand, in [20] (extended later in [21, 22]), although routing is taken into account, the methodology of solving the problem is different and does not guaranty the optimality of solutions. What is more, in both cases perfect IC is assumed and overheard transmissions are not taken into account as far as IC is concerned.

\section{Optimization Model}

3.1. Notation. In order to formally present the problem, we introduce the following notation.

Indices

$e$ : Mesh link

$t$ : Time slot 
$m: \mathrm{MCS}$

$v, w:$ Mesh nodes

$d$ : Demand

$(v, w)$ : Mesh link from mesh node $v$ to mesh node $w$

$a(e)$ : Mesh node that is a source of mesh link $e$

$b(e)$ : Mesh node that is a sink of mesh link $e$.

Sets
$\mathscr{V}:$ Set of mesh nodes
$\mathscr{E}:$ Set of mesh links
$\delta^{+}(e)$ : Set of mesh links leaving mesh node $e$
$\delta^{-}(e)$ : Set of mesh links entering mesh node $e$
$\mathscr{D}$ : Set of demands
$\mathscr{P}_{d}$ : Set of mesh links that form a path for demand $d$
$\mathscr{M}$ : Set of MCSs
$\mathscr{T}$ : Set of time slots.

\section{Constants}

$B^{m}:$ Bit rate of MCS $m$

$J_{v e}^{m}: 1$ if mesh node $v$ can jam a transmission at mesh link $e$ that uses MCS $m$; 0 otherwise; notice that $J_{v e}^{m}=$ 0 when $a(e)=v$

$T_{e}^{m}: 1$ if mesh linke can be successfully used with MCS $m ; 0$ otherwise.

\section{Variables}

$Y_{e t}^{m}: 1$ if mesh link $e$ is transmitting in time slot $t$ using MCS $m ; 0$ otherwise

$X_{v t}: 1$ if mesh node $v$ is transmitting in time slot $t ; 0$ otherwise

$y_{e t v}^{m}: 1$ if mesh link $e$ is transmitting in time slot $t$ using MCS $m$ and the data is decoded in mesh node $v ; 0$ otherwise; notice that the data can be decoded not only by $b(e)$

$x_{v t m}^{m}: 1$ if mesh node $v$ is transmitting in time slot $t$ using MCS $m$ but mesh node $w$ is canceling the interference; 0 otherwise

$l_{v w}$ : Average transmission bit rate sent to mesh node $v$ overheard by mesh node $w$

$c_{e}$ : Average transmission bit rate at mesh link $e$

$h_{d}$ : Average transmission bit rate for demand $d$

$h_{\min }$ : Average transmission bit rate for the worst served demand.

In order to use the above notation, the defined sets and constants have to be relatively stable and possible to obtain. Therefore, the following requirements have to be met. (i) $\mathscr{V}$ is known and does not change. When failures are not considered, the requirement is met.

(ii) $\mathscr{E}$ is known and does not change. When propagating conditions are relatively stable and nodes do not move, the requirement is met.

(iii) $\delta^{+}(e)$ is known and does not change. See above.

(iv) $\mathscr{D}$ is known and does not change. When a network consists of a number of MPPs and all other nodes are connected to them using static paths, the requirement is met.

(v) $\mathscr{P}_{d}$ serving a demand $d$ (for all $d \in \mathscr{D}$ ) does not change. When a routing is static, the requirement is met.

(vi) $\mathscr{M}$ is known and does not change. The requirement is always met.

(vii) $\mathscr{T}$ is known and does not change. When a network is synchronized, the requirement is met. We assume that a number of possible time slots are given.

(viii) $B^{m}$ are known and do not change. The requirement is always met.

(ix) $J_{v e}^{m}$ are known and do not change. The requirement needs a detailed discussion; see below.

(x) $T_{e}^{m}$ are known and do not change. In our research we compute constants $T_{e}^{m}$ and $J_{v e}^{m}$ using a mechanism described below. When properly used, the mechanisms guarantee that the requirements concerning $T_{e}^{m}$ and $J_{v e}^{m}$ are met.

An approach used to compute constants $T_{e}^{m}$ and $J_{v e}^{m}$ is similar to the one presented in [11]. In order to explain it in details the following notation is necessary.

$N$ : Noise level

$S_{m}:$ SINR threshold for MCS $m$

$P_{v w}$ : Power received by mesh node $w$ when mesh node $v$ is transmitting.

The notation above does not appear in the final formulation of the problem and is solely used in order to define constants $T_{e}^{m}$ and $J_{v e}^{m}$. The former constant is defined by (1), while the latter by (2). The equations guarantee that a given MCS can be used only if power of a signal of interest received at a node is greater than the noise and considered interferences. It is worth to mention that constants $S_{m}$ are in linear scale here and not logarithmic:

$$
\begin{gathered}
T_{e}^{m}= \begin{cases}1 & \text { if } \frac{P_{a(e) b(e)}}{N} \geq S_{m} \\
0 & \text { if } \frac{P_{a(e) b(e)}}{N}<S_{m},\end{cases} \\
J_{v e}^{m}= \begin{cases}1 & \text { if } \frac{P_{a(e) b(e)}}{N+P_{v b(e)}}<S_{m} \\
0 & \text { if } \frac{P_{a(e) b(e)}}{N+P_{v b(e)}} \geq S_{m} .\end{cases}
\end{gathered}
$$


If we assume that mesh nodes do not move and propagating conditions are relatively stable, then $N$ and $P_{v w}$ can be treated as constants in both (1) and (2). As $S_{m}$ is also a constant, therefore $T_{e}^{m}$ and $J_{v e}^{m}$ can also be treated as constants.

Finally, we assume that a kind of OSPF routing is used. The assumption is irrelevant from the point of view of the notation. However, in our model we require that a path used by a packet before reaching a given node is irrelevant. The requirement is met when there is exactly one path to each node; thus it can be met when a kind of OSPF routing is used in a network.

To summarize, in order to use the model presented in this paper, the following conditions have to be assumed.

(i) Failures are not considered.

(ii) Mesh nodes do not move.

(iii) Propagating conditions are relatively stable.

(iv) Network consists of a number of MPPs and all other nodes are connected to them using static paths.

(v) Routing is static.

(vi) Network is synchronized.

(vii) Kind of OSPF routing is used.

3.2. MIP Model. When the conditions presented above are assumed, we can formally state the problem in the form of formulations $(3 \mathrm{a})-(3 \mathrm{~m})$ below. It is a compact mixed integer-linear programming formulation that has proved to be tractable for network consisting of tens of nodes:

maximize $h_{\min }$

subject to

$$
\begin{aligned}
& \sum_{m \in \mathscr{M}} \sum_{e \in \delta^{+}(v)} Y_{e t}^{m}=X_{v t} \quad \forall v \in \mathscr{V}, \forall t \in \mathscr{T}, \\
& y_{e t v}^{m} \leq Y_{e t}^{m} \quad \forall e \in \mathscr{E}, \forall t \in \mathscr{T}, \forall v \in \mathscr{V}, \quad \forall m \in \mathscr{M}, \\
& l_{v w}=\frac{1}{|\mathscr{T}|} \sum_{m \in \mathscr{M}} B^{m} \sum_{t \in \mathscr{T}} \sum_{e: b(e)=v} y_{e t w}^{m} \quad \forall v, w \in \mathscr{V}, \\
& l_{v w} \geq \frac{1}{|\mathscr{T}|} \sum_{m \in \mathscr{M}} B^{m} \sum_{t \in \mathscr{T}} x_{v t w}^{m} \quad \forall v, w \in \mathscr{V}, \\
& x_{v t w}^{m} \leq \sum_{e \in \delta^{+}(v)} Y_{e t}^{m} \quad \forall v, w \in \mathscr{V}, \forall m \in \mathscr{M}, \forall t \in \mathscr{T}, \\
& y_{e t w}^{m}+X_{v t}-\sum_{m^{\prime} \in \mathscr{M}} x_{v t w}^{m^{\prime}} \leq 2-J_{v(a(e), w)}^{m} \quad \forall e \in \mathscr{E},
\end{aligned}
$$

$\forall t \in \mathscr{T}, \quad \forall m \in \mathscr{M}, \quad \forall v, w \in \mathscr{V}$,

$$
\begin{aligned}
& y_{e t v}^{m} \leq T_{(a(e), v)}^{m} \quad \forall e \in \mathscr{E}, \forall t \in \mathscr{T}, \forall m \in \mathscr{M}, \forall v \in \mathscr{V}, \\
& c_{e}=\frac{1}{|\mathscr{T}|} \sum_{m \in \mathscr{M}} B^{m} \sum_{t \in \mathscr{T}}\left(y_{e t b(e)}^{m}-x_{a(e) t b(e)}^{m}\right) \quad \forall e \in \mathscr{C}, \\
& x_{v t v}^{m}=0 \quad \forall v \in \mathscr{V}, \forall t \in \mathscr{T}, \forall m \in \mathscr{M}, \\
& \sum_{d: e \in \mathscr{P}_{d}} h_{d} \leq c_{e} \quad \forall e \in \mathscr{E}, \\
& h_{\min } \leq h_{d} \quad \forall d \in \mathscr{D}, \\
& X_{v t}, Y_{e t}^{m}, y_{e t v}^{m}, x_{v t w}^{m} \in\{0,1\} \\
& l_{v w}, c_{e}, h_{d}, h_{\min } \geq 0
\end{aligned}
$$

In our research we use the max-min cost function [23]; thus the objective of $(3 \mathrm{a})-(3 \mathrm{~m})$ is to maximize the minimum flow $h_{\min }$ to the worst served MP. Constraint (3a) assures that a node during one time slot can transmit only on one link using only one MCS. Constraint (3b) prevents nodes from decoding data that are not transmitted. In other words, only packets that have been sent can be decoded. The purpose of (3c) is to calculate the average transmission bit rate sent to $b(e)$ ( $w$ in the next constraint) and decoded by $v$. The computed value is an upper bound for the average transmission bit rate sent from $w$ and canceled in $v$. The upper bound is enforced by (3d). Obviously the interference cancelation can take place only if a transmission to be canceled takes place. This condition is enforced by (3e). The next constraint, that is, (3f), assures that a transmission can be successfully decoded only if it is not jammed by other transmissions. Notice that transmissions that have been earlier overheard and currently are canceled cannot jam concurrent transmissions. Constraint (3g) assures that only MCSs satisfying SNR thresholds are used, while (3h) calculates the average useful transmission bit rate at each link. Notice that canceled transmissions do not count towards the average useful transmission bit rate. Constraint (3i) disallows MPs to cancel self-interferences. It is worth noticing that some work on canceling self-interferences exists and a fullduplex has even been implemented in practice [24]. However, it required a special technique called antenna cancelation; thus in our research we assume that the self-interferences cancelation is impracticable and is not being used. Constraint (3j) divides available capacity of a network between different traffic demands, and (3k) assures that the division is fair; average throughput of the worst served demand is maximized. Finally, constraints (3l) and (3m) are responsible for binarity and nonnegativity of the variables.

3.3. Valid Inequalities. The presented MIP model is relatively complicated and solving it requires significant computation effort. In order to increase its applicability, we present three practical valid inequalities. They are not indispensable from the point of view of the correctness of the model. However, they tight a polytop of a relaxed problem, thus making branch-and-bound approach work more efficiently:

$$
\sum_{t \in \mathscr{T}} \sum_{m \in \mathscr{M}}\left(y_{e t b(e)}^{m}-x_{a(e) t b(e)}^{m}\right) \geq 1 \quad \forall e \in \mathscr{P}_{d}, \forall d \in \mathscr{D},
$$


TABLE 1: Modulation and coding schemes.

\begin{tabular}{lcccc}
\hline Modulation & Coding & Throughput & SINR [log.] & SINR [lin.] \\
\hline BPSK & $1 / 2$ & $6.0 \mathrm{Mbps}$ & $3.48 \mathrm{~dB}$ & 2.23 \\
QPSK & $1 / 2$ & $12.0 \mathrm{Mbps}$ & $6.63 \mathrm{~dB}$ & 4.6 \\
16-QAM & $1 / 2$ & $24.0 \mathrm{Mbps}$ & $12.76 \mathrm{~dB}$ & 18.88 \\
64-QAM & $1 / 2$ & $48.0 \mathrm{Mbps}$ & $20.29 \mathrm{~dB}$ & 106.9 \\
\hline
\end{tabular}

$X_{v t}+\sum_{e \in \mathscr{E}} \sum_{m \in \mathscr{M}}\left(y_{e t v}^{m}-x_{a(e) t v}^{m}\right) \leq 1 \quad \forall v \in \mathscr{V}, \forall t \in \mathscr{T}$

$\sum_{e \in \delta^{-}(v)} \sum_{t^{\prime} \in \mathscr{T}} \sum_{m^{\prime} \in \mathscr{M}} y_{e t^{\prime} v}^{m^{\prime}} \geq x_{v t w}^{m} \quad \forall v, w \in \mathscr{V}, \forall t \in \mathscr{T}, \forall m \in \mathscr{M}$.

The first valid inequality (4a) guarantees that on each mesh link that is used by at least one demand there is a transmission scheduled during at least one time slot. What is more, during that time slot not only the transmission has to occur but also it cannot be subject to interference cancelation at the receiving end of the mesh link. The inequality limits the number of possibilities of dividing time slots into smaller parts while solving relaxed versions of the problem during the $\mathrm{B} \& \mathrm{~B}$ process. Notice that this inequality is formally not a valid inequality, as it cuts feasible solutions of cost 0 . However, from the practical point of view, those solutions are useless. Therefore, we decided to treat this inequality as valid.

The second valid inequality (4b) guarantees that a mesh node either transmits or receives data from aother mesh node. The inequality disallows a number of relaxed solutions when a mesh node receives and decodes a number of "half" transmissions and simultaneously "half" transmits itself; that is, $y_{\text {etv }}^{m}=0.5$ and $X_{v t}=0.5$ in constraint (3f).

The last valid inequality (4c) guarantees that if a mesh node performs interference cancelation during at least one time slot, then it had to overheard a transmission to be canceled during at least one other time slot. The inequality introduces tight connections between binary variables $y_{e t^{\prime} v}^{m^{\prime}}$ and $x_{v t w}^{m}$. In model (3a)- $(3 \mathrm{~m})$ the variables were related using variable $l_{v w}$ and constraints $(3 \mathrm{c})$ and $(3 \mathrm{~d})$.

\section{Numerical Results}

We tested numerically the optimization model presented in Section 3 using CPLEX 12.5 set up on Intel Core 2 Quad CPU Q6600 2.40 GHz with 4.00 GB RAM. We used 50 randomly generated network topologies for each test case from Table 2. All nodes of the networks were randomly located on a square. We followed [25] and assumed the path loss exponent of 4 while calculating power received at each station and establishing mesh links. Notice that the topology and the path loss exponent allow us to calculate both $T_{e}^{m}$ and $J_{v e}^{m}$. Each MP is routed to the nearest MPP using the shortest path with respect to weights of links. We set the weight of link $e$ to the inverse of $P_{a(e) b(e)}$. Each link uses an available (satisfying the SINR constraint) MCS from Table 1 with maximal $B^{m}$. In order to minimize a number of infeasible instances, locations of nodes were scaled in such a way that the longest utilized
TABLE 2: Considered network topologies.

\begin{tabular}{lccc}
\hline Topology & MPPs & MPs & Avg. mesh links \\
\hline N6 & 5 & 1 & 15.0 \\
N8 & 7 & 1 & 25.0 \\
N10 & 8 & 2 & 32.5 \\
N12 & 10 & 2 & 42.3 \\
N14 & 11 & 3 & 54.0 \\
N16 & 13 & 3 & 61.4 \\
N18 & 15 & 3 & 71.4 \\
N20 & 16 & 4 & 83.5 \\
N22 & 18 & 4 & 97.5 \\
N24 & 19 & 5 & 104.2 \\
N26 & 21 & 5 & 114.1 \\
N28 & 23 & 5 & 131.8 \\
N30 & 24 & 6 & 143.4 \\
\hline
\end{tabular}

link in a network was of a maximal length allowing for a successful transmission using the least demanding MCS.

Numerical results are presented in Table 3. The first column contains topology identifiers. They inform which test case from Table 2 is under consideration. The second column contains the number of time slots in a test case. The following six columns contain efficiency specific information. For each case we gathered mean running time of the MIP solver, a number of timeouts (the time limit was set to ten minutes), and a mean number of $B \& B$ nodes entered, for both a case with valid inequalities of Section 3.3 and a case without the valid inequalities. The last two columns justify usage of IC in WMN. The first of them contains a percentage of cases which can be improved by employing IC, while the second contains an average obtained gain for those cases. It is expressed in percents and understood as $(B-A) / A$ times $100 \%$, where $A$ is $h_{\min }$ for a test case that does not use IC and $B$ is $h_{\min }$ for a test case taking advantage of IC.

The numerical results prove the expected complexity of the problem. Running times show a limited tractability of the presented MIP model. However, the limited tractability is accompanied by considerable gains in terms of network capacity. It is worth mentioning that the max-min cost function used in our research is one of the most restrictive and in many cases it is impossible to obtain better results due to one very inconveniently located mesh node. In those cases, although the max-min gain is equal to zero, using IC techniques still can increase throughput for better served mesh nodes.

As for the usage of the valid inequalities of Section 3.3, it almost always decreases a number of visited B\&B nodes. However, it not always assures that a problem will be solved faster. In fact, as can be seen in Figure 3, it is usually profitable to use the valid inequalities for cases with a small number of time slots. However, when the number of time slots increases, then the valid inequalities lose their importance.

Running times presented in Figure 3 may create an impression that the model is useless for larger networks. Additionally taking into account the information about timeouts presented in Table 3, one may claim that with so 
TABLE 3: Numerical results.

\begin{tabular}{|c|c|c|c|c|c|c|c|c|c|}
\hline \multirow{2}{*}{ Top. } & \multirow{2}{*}{$|\mathfrak{T}|$} & \multicolumn{3}{|c|}{ Without valid inequalities } & \multicolumn{3}{|c|}{ With valid inequalities } & \multirow{2}{*}{ Improved [\%] } & \multirow{2}{*}{ Gain [\%] } \\
\hline & & Time $[\mathrm{s}]$ & \#EOT & $\# \mathrm{~B} \& \mathrm{~B}$ & Time $[\mathrm{s}]$ & \#EOT & $\# \mathrm{~B} \& \mathrm{~B}$ & & \\
\hline N6 & 8 & 0.22 & 0 & 7.48 & 0.3 & 0 & 0.6 & 4.0 & 46.7 \\
\hline N6 & 12 & 0.3 & 0 & 3.42 & 0.42 & 0 & 43.2 & 6.0 & 23.3 \\
\hline N8 & 8 & 0.56 & 0 & 36.54 & 0.76 & 0 & 18.2 & 18.0 & 35.6 \\
\hline N8 & 12 & 0.74 & 0 & 91.64 & 1.32 & 0 & 15.2 & 12.0 & 28.9 \\
\hline N10 & 8 & 0.86 & 0 & 60.16 & 1.66 & 0 & 32.0 & 14.0 & 53.8 \\
\hline N10 & 12 & 13.24 & 1 & 693.38 & 4.46 & 0 & 131.1 & 16.0 & 45.5 \\
\hline N12 & 8 & 1.64 & 0 & 143.02 & 3.4 & 0 & 42.1 & 20.0 & 56.1 \\
\hline N12 & 12 & 19.64 & 1 & 1093.7 & 22.76 & 1 & 943.4 & 24.0 & 28.3 \\
\hline N14 & 8 & 1.9 & 0 & 193.82 & 2.36 & 0 & 45.5 & 16.0 & 73.1 \\
\hline N14 & 12 & 2.98 & 0 & 199.96 & 7.3 & 0 & 242.1 & 12.0 & 22.1 \\
\hline N16 & 8 & 15.68 & 1 & 616.06 & 6.92 & 0 & 73.3 & 12.0 & 45.7 \\
\hline N16 & 12 & 16.26 & 0 & 471.9 & 29.22 & 0 & 532.2 & 28.0 & 25.9 \\
\hline N18 & 8 & 17.84 & 1 & 3065.92 & 8.34 & 0 & 209.1 & 20.0 & 45.5 \\
\hline N18 & 12 & 33.22 & 2 & 2278.5 & 28.4 & 1 & 517.0 & 36.0 & 25.3 \\
\hline N20 & 8 & 54.88 & 4 & 3142.44 & 31.14 & 1 & 280.3 & 8.0 & 22.0 \\
\hline $\mathrm{N} 20$ & 12 & 78.56 & 5 & 2479.86 & 76.32 & 4 & 990.6 & 22.0 & 30.9 \\
\hline $\mathrm{N} 22$ & 8 & 36.04 & 2 & 4182.44 & 20.26 & 0 & 334.3 & 22.0 & 47.1 \\
\hline $\mathrm{N} 22$ & 12 & 98.4 & 5 & 7991.82 & 95.18 & 4 & 1196.5 & 28.0 & 29.2 \\
\hline $\mathrm{N} 24$ & 8 & 58.22 & 4 & 5092 & 18.36 & 0 & 308.6 & 20.0 & 71.9 \\
\hline $\mathrm{N} 24$ & 12 & 81.1 & 5 & 3892.46 & 84.62 & 5 & 1651.6 & 24.0 & 30.5 \\
\hline N26 & 8 & 63.36 & 4 & 11441.86 & 26.4 & 0 & 581.9 & 18.0 & 53.9 \\
\hline N26 & 12 & 110.48 & 6 & 5597.08 & 127.74 & 6 & 2314.8 & 32.0 & 25.8 \\
\hline N28 & 8 & 64.5 & 3 & 4092 & 46 & 0 & 802.3 & 16.0 & 41.1 \\
\hline N28 & 12 & 188.84 & 12 & 7933.38 & 207.88 & 13 & 3962.1 & 34.0 & 32.3 \\
\hline N30 & 8 & 157.5 & 10 & 16574.44 & 55.44 & 1 & 868.8 & 20.0 & 54.7 \\
\hline N30 & 12 & 192.08 & 9 & 13423.08 & 197.5 & 11 & 3167.3 & 26.0 & 28.1 \\
\hline \multicolumn{2}{|c|}{ Average } & 50.35 & 2.88 & 3646.1 & 42.48 & 1.81 & 742.5 & 19.54 & 39.35 \\
\hline
\end{tabular}

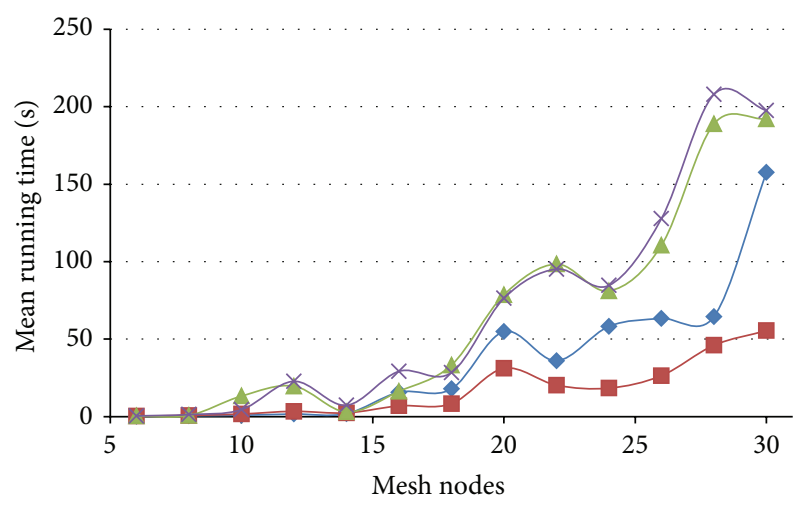

$8 \mathrm{TS}$, w/o valid inequalities $-12 \mathrm{TS}$, w/o valid inequalities $8 \mathrm{TS}$, with valid inequalities $\leftarrow 12 \mathrm{TS}$, with valid inequalities

Figure 3: Running times.

many unsolved test cases the model is impractical. However, notice that a timeout does not mean that the MIP solver has not returned any solution for a test case. A timeout only means that the MIP solver was not able to prove an optimality

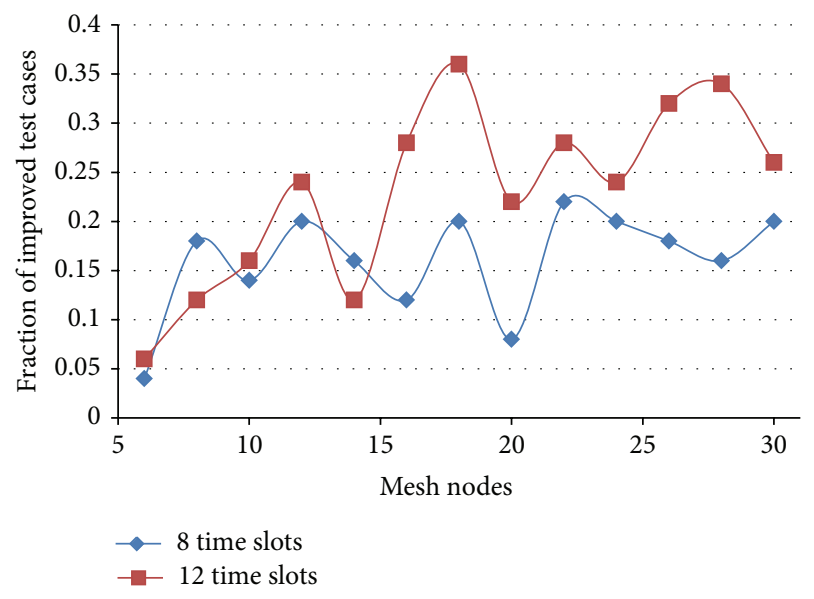

Figure 4: Fraction of improved test cases.

of a returned solution, which still in many cases was better than a solution obtained when IC was not considered.

Finally, our results show an interesting impact of increasing the number of time slots on the problem. As expected, increasing a number of time slots complicates the problem 


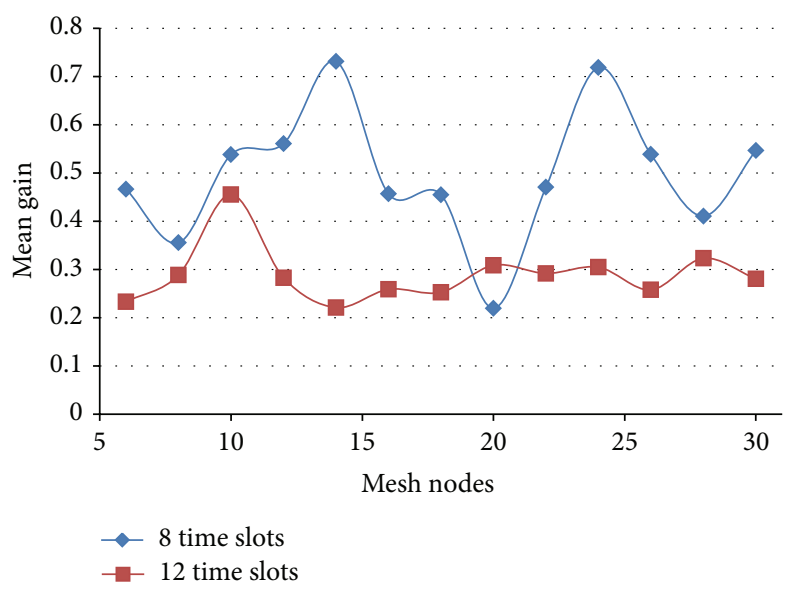

FIGURE 5: Mean gain.

making it more difficult to solve. This fact is clearly seen in Figure 3. However, an impact of increasing the number of time slots on efficiency of IC is not so straightforward. On one hand, as seen in Figure 4, the more the time slots are available, the bigger the chance that using IC can be profitable. On the other hand, as seen in Figure 5, the more the time slots are available, the smaller the gain IC can bring. Such a behavior is a result of an increased elasticity brought by an increased number of available time slots. The increased elasticity extends a feasibility region of our problem making it easier to find better solutions than those available in a case when IC is disallowed. On the other hand, with increased elasticity, all the changes in a solution result in much smaller impact on the global objective function, thus making possible gains smaller.

\section{Conclusion}

In the paper we show that the idea of interference cancelation can significantly increase the capacity of a wireless mesh network even when the max-min objective function is taken into account, and the interference cancelation can be applied only to signals that have been overheard in the past. In order to approximate possible advantages of using interference cancelation in the considered conditions, we presented a novel MIP model that allows for calculating perfect scheduling and maximal throughput in a network. We compare our results with results obtained for cases when the interference cancelation mechanisms are disabled. Our results show that using interference cancelation mechanisms for signals that have been overheard in the past increases a network throughput by $40 \%$ on average in approximately $20 \%$ of test cases.

The work presented in this paper can be extended in many different directions. One of possible extensions is to consider the full interference model instead of the used simplified interference model. Another interesting research direction is to present the problem using a noncompact formulation based on the idea of compatible sets (see $[11,17])$ that allows for an indirect consideration of an infinite number of time slots. Notice that both of the suggested possible extensions cannot be directly incorporated into our MIP model and require deeper studies.

\section{Conflict of Interests}

The author declares that there is no conflict of interests regarding the publication of this paper.

\section{Acknowledgment}

The work presented in this paper has been funded by National Science Center (Poland) under Grant 2011/01/B/ST7/02967 "integer programming models for joint optimization of link capacity assignment, transmission scheduling, and routing in fair multicommodity flow networks."

\section{References}

[1] "Bay area wireless users group," http://www.bawug.com/.

[2] "Seattle wireless," http://www.seattlewireless.net/.

[3] I. F. Akyildiz and X. Wang, "A survey on wireless mesh networks," IEEE Communications Magazine, vol. 43, no. 9, pp. S23-S30, 2005.

[4] R. Bruno, M. Conti, and E. Gregori, "Mesh networks: commodity multihop ad hoc networks," IEEE Communications Magazine, vol. 43, no. 3, pp. 123-131, 2005.

[5] IEEE Standard for Information Technology, "Telecommunications and information exchange between systems-local and metropolitan area networks-specific requirements. Part 11: wireless LAN Medium Access Control (MAC) and Physical Layer (PHY) specifications amendment 5: enhancements for higher throughput," IEEE Std 802.11n-2009 (Amendment to IEEE Std $802.11-2007$ As Amended by IEEE Std 802.11k-2008, IEEE Std 802.11r-2008, IEEE Std 802.11y-2008, and IEEE Std 802.11w-2009), 2009.

[6] J. G. Andrews, "Interference cancellation for cellular systems: a contemporary overview," IEEE Wireless Communications, vol. 12, no. 2, pp. 19-29, 2005.

[7] N. I. Miridakis and D. D. Vergados, "A survey on the successive interference cancellation performance for single-antenna and multiple-antenna OFDM systems," IEEE Communications Surveys \& Tutorials, vol. 15, no. 1, pp. 312-335, 2013.

[8] D. Tse and P. Viswanath, Fundamentals of Wireless Communication, Cambridge University Press, New York, NY, USA, 2005.

[9] V. R. Cadambe and S. A. Jafar, "Interference alignment and degrees of freedom of the K-user interference channel," IEEE Transactions on Information Theory, vol. 54, no. 8, pp. 34253441, 2008.

[10] S. Gollakota, S. D. Perli, and D. Katabi, "Interference alignment and cancellation," ACM SIGCOMM Computer Communication Review, vol. 39, no. 4, pp. 159-170, 2009.

[11] M. Pióro, M. Zotkiewicz, B. Staehle, D. Staehle, and D. Yuan, "On max-min fair flow optimization in wireless mesh networks," Ad Hoc Networks, vol. 13, part A, pp. 134-152, 2014.

[12] D. Halperin, T. Anderson, and D. Wetherall, "Taking the sting out of carrier sense: interference cancellation for wireless LANs," in Proceedings of the 14th ACM Annual International Conference on Mobile Computing and Networking (MobiCom '08), pp. 339-350, San Francisco, Calif, USA, September 2008. 
[13] D. Yuan, V. Angelakis, L. Chen, E. Karipidis, and E. G. Larsson, "On optimal link activation with interference cancelation in wireless networking," IEEE Transactions on Vehicular Technology, vol. 62, no. 2, pp. 939-945, 2013.

[14] B. Bandemer, Q. Li, X. E. Lin, and A. Paulraj, "Overhearingbased interference cancellation for relay networks," in Proceedings of the 70th IEEE Vehicular Technology Conference Fall (VTC-Fall '09), pp. 1-5, Anchorage, Alaska, USA, September 2009.

[15] S. Zhang, S.-C. Liew, and H. Wang, "Blind known interference cancellation," IEEE Journal on Selected Areas in Communications, vol. 31, no. 8, pp. 1572-1582, 2013.

[16] L. E. Li, R. Alimi, D. Shen, H. Viswanathan, and Y. R. Yang, "A general algorithm for interference alignment and cancellation in wireless networks," in Proceedings of the 29th IEEE International Conference on Computer Communications (INFOCOM '10), pp. 1-9, San Diego, Calif, USA, March 2010.

[17] A. Capone, G. Carello, I. Filippini, S. Gualandi, and F. Malucelli, "Routing, scheduling and channel assignment in Wireless Mesh Networks: optimization models and algorithms," Ad Hoc Networks, vol. 8, no. 6, pp. 545-563, 2010.

[18] M. Pióro and D. Medhi, Routing, Flow, and Capacity Design in Communication and Computer Networks, Morgan Kaufmann, 2004.

[19] V. Angelakis, L. Chen, and D. Yuan, "Optimal and collaborative rate selection for interference cancellation in wireless networks," IEEE Communications Letters, vol. 15, no. 8, pp. 819-821, 2011.

[20] S. Lv, W. Zhuang, X. Wang, and X. Zhou, "Scheduling in wireless ad hoc networks with successive interference cancellation," in Proceedings of the 30th IEEE International Conference on Computer Communications (INFOCOM '11), pp. 1287-1295, Shanghai, China, April 2011.

[21] S. Lv, W. Zhuang, X. Wang, C. Liu, and X. Zhou, "Maximizing capacity in the SINR model in wireless networks with successive interference cancellation," in Proceedings of the IEEE International Conference on Communications (ICC '11), pp. 1-6, Kyoto, Japan, June 2011.

[22] S. Lv, W. Zhuang, X. Wang, and X. Zhou, "Link scheduling in wireless networks with successive interference cancellation," Computer Networks, vol. 55, no. 13, pp. 2929-2941, 2011.

[23] W. Ogryczak, M. Pióro, and A. Tomaszewski, "Telecommunications network design and max-min optimization problem," Journal of Telecommunications and Information Technology, vol. 3, pp. 43-56, 2005.

[24] J. Choi II, M. Jain, K. Srinivasan, P. Levis, and S. Katti, "Achieving single channel, full duplex wireless communication," in Proceedings of the 16th ACM Annual Conference on Mobile Computing and Networking (MobiCom '10), pp. 1-12, September 2010.

[25] A. Goldsmith, Wireless Communications, Cambridge University Press, 2005. 


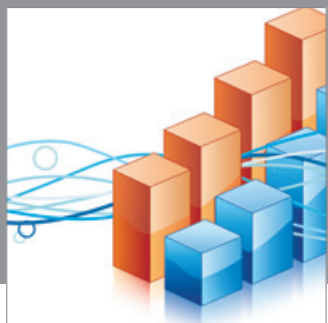

Advances in

Operations Research

mansans

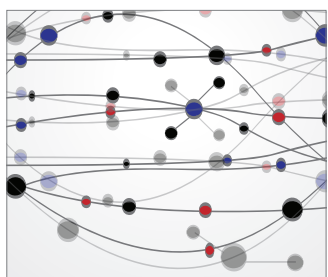

The Scientific World Journal
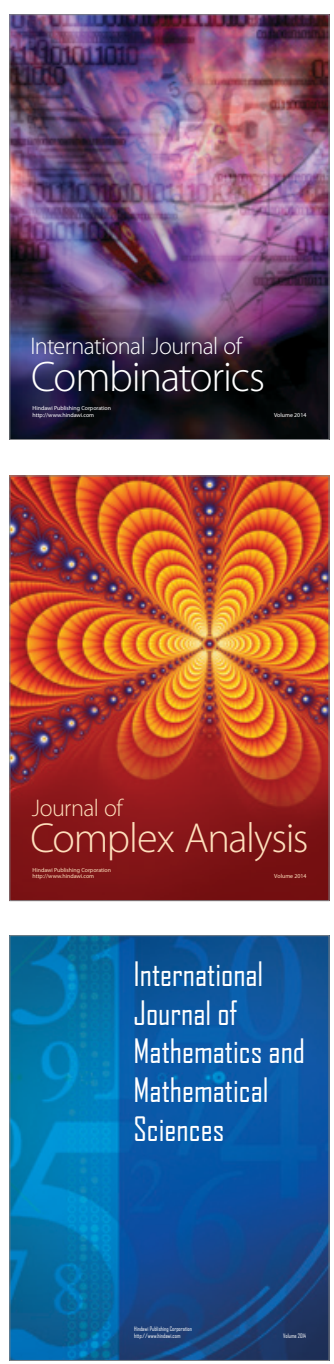
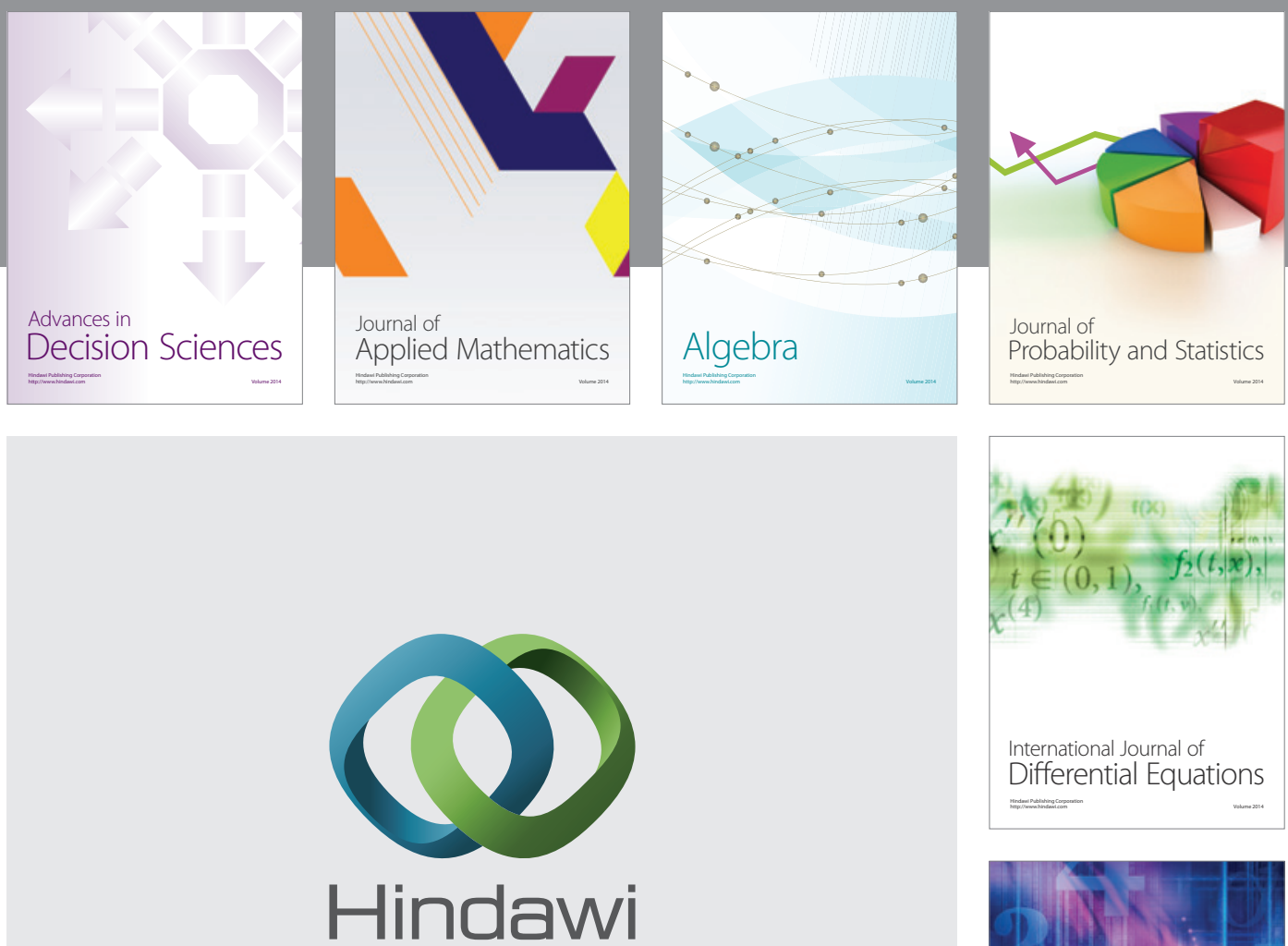

Submit your manuscripts at http://www.hindawi.com
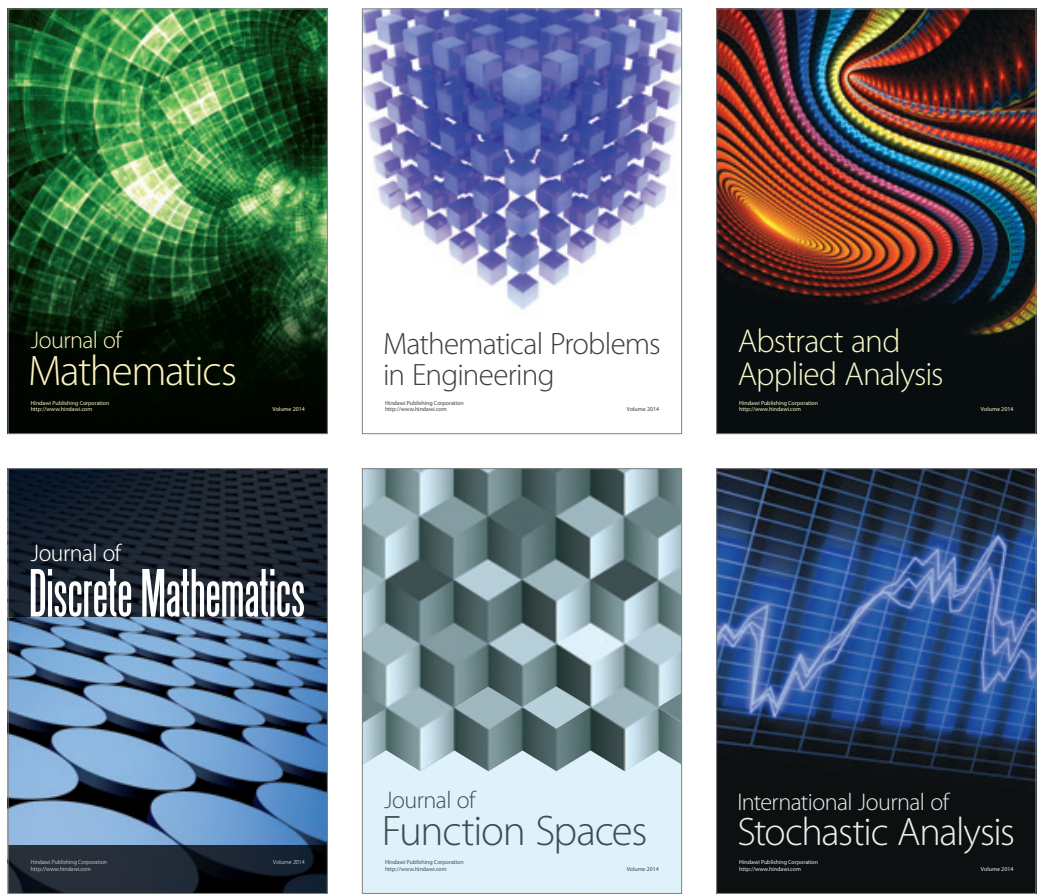

Journal of

Function Spaces

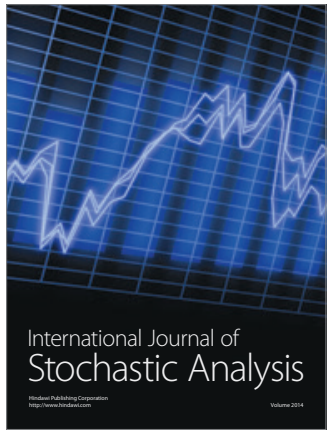

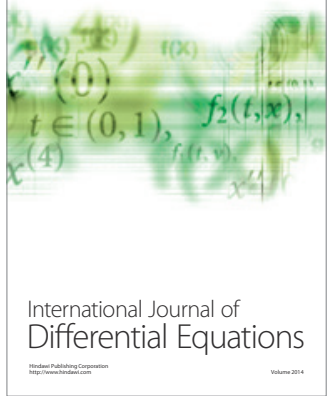
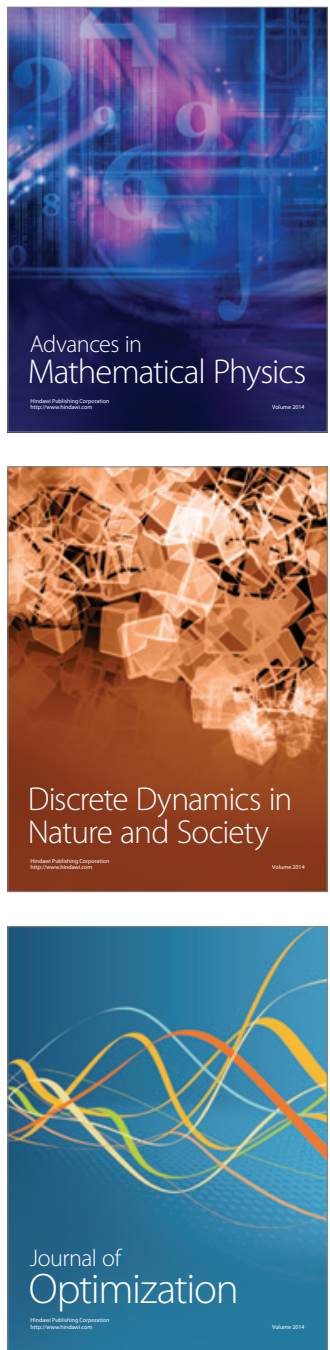